\title{
First-principles calculation of the longitudinal phonon in the surface-normal direction of a zirconium(0001) slab: Localization mode at the subsurface
}

\author{
Masahiro Yamamoto \\ Institute of Atomic Energy, Kyoto University, Uji, Kyoto 611, Japan \\ C. T. Chan \\ Physics Department, Hong Kong University of Science and Technology, Clear Water Bay, Hong Kong \\ and Ames Laboratory-United States Department of Energy, Department of Physics and Astronomy, \\ Iowa State University, Ames, Iowa 50011 \\ K. M. Ho \\ Ames Laboratory-United States Department of Energy and Department of Physics and Astronomy, \\ Iowa State University, Ames, Iowa 50011 \\ Mitsunori Kurahashi and Shizuo Naito \\ Institute of Atomic Energy, Kyoto University, Uji, Kyoto 611, Japan \\ (Received 24 July 1995)
}

\begin{abstract}
The energies and amplitudes of the longitudinal vibrational mode in the surface-normal [0001] direction of the fully relaxed zirconium slab with a (0001) surface are calculated with force constants determined from first-principles total-energy and force calculations. A subsurface vibrational mode with frequency above the bulk continuum is found. We attribute the origin of this mode to the hardness of the force constant between the surface and the second layer.
\end{abstract}

Most transition-metal surfaces exhibit relaxations; i.e., the interlayer spacing between the first and the second layer is reduced and an oscillatory relaxation pattern of the inner layers is usually observed. ${ }^{1}$ Since the redistribution of charges on the surface causes the relaxation of the top few layers and the change of the interlayer force constants, the vibrational mode in the surface-normal direction is expected to be changed with the relaxation.

Surface lattice dynamics has been studied experimentally by high-resolution electron energy-loss spectroscopy (HREELS) and helium atom scattering (HAS) methods. ${ }^{2,3}$ In the HAS method, the vibrational motion of the surface layer is detected and the change of the vibrational behavior in the surface-normal direction has been obtained indirectly. The information of the subsurface vibrational motion can be obtained directly by HREELS, but its energy resolution (ca. 7 $\mathrm{meV}$ ) is much less than that of the HAS method (ca. 0.2 $\mathrm{meV})$. In the case of group IV transition metals such as titanium, zirconium, and hafnium, no detailed surface phonon dispersion has been reported. The hydrogen contamination of the surface may change the surface relaxation and also the dynamical properties of the surfaces. ${ }^{4}$ The change of the vibrational motion with the surface normal direction has been investigated with Debye-Waller factor analysis for lowenergy electron diffraction (LEED) from the $\mathrm{Ti}(0001)$ surface $^{5}$ and electron energy-loss fine-structure (EELFS) measurements from a polycrystalline titanium surface. ${ }^{6}$ The Debye temperature, which is a measure of the rigidity of the lattice, is monotonically decreased with increase in the surface properties of the detected vibrational motion. However, these analyses give the averaged information of the dynamical properties over the several surface layers.
First-principles total-energy and force calculations have been shown to give an accurate description of the surface phonon dynamics. ${ }^{7}$ However, the phonon dynamics in the surface-normal direction of the slab has not been investigated in detail. In the present study the vibrational properties of the zirconium (0001) slab are investigated by using the results of the first-principles total-energy and force calculations for this surface.

The first-principles calculations have been carried out using a pseudopotential method within the framework of the density-functional theory. The Hedin-Lundqvist form of exchange-correlation functionals has been used. The Bloch wave functions are expanded in a mixed basis of plane waves and numerical localized wave functions centered on atomic sites. We used a plane-wave basis with kinetic energy up to 15 Ry. Details of the total-energy and force calculations based on the Hellmann-Feynman theorem are given in Ref. 8.

To check the accuracy of the frozen phonon calculation we calculate the vibrational energies of the zone-center phonon of the longitudinal-optic (LO) and transverse-optic (TO) modes for hcp zirconium. The energies and forces are calculated for the configurations with atomic displacements as shown in Figs. 1(a) and 1(b), and the zone-center TO and LO phonon energies are evaluated in the limit of zero atomic displacement. The energies of the zone-center LO and TO phonons are found to be 20.5 and $9.0 \mathrm{meV}$ from the totalenergy calculation, respectively, and 21.8 and $9.7 \mathrm{meV}$ from the force calculation, respectively. These values are in agreement with the experimental results from the neutron inelastic scattering by Stassis et al. ${ }^{9}$ The phonon energies at $5.5 \mathrm{~K}$ were found to be 17.5 and $11.0 \mathrm{meV}$ for the $\mathrm{LO}$ and $\mathrm{TO}$ modes, respectively. The calculated values (closed circles) 


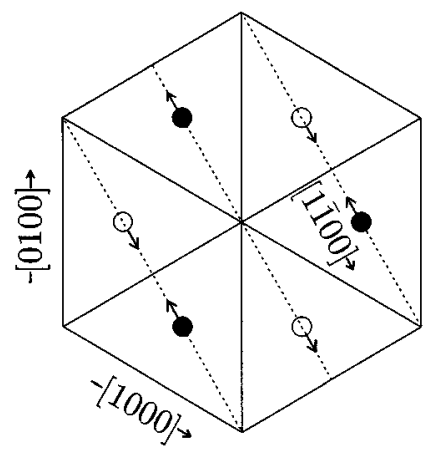

(a)

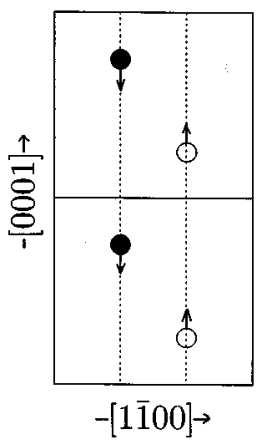

(b)
FIG. 1. Atomic configuration for the frozen phonon calculation of the transverse-optic (TO) (a) and the longitudinal-optic (LO) (b) phonon in the [0001] direction of hcp zirconium.

and the phonon dispersion relations of the hep zirconium in the [0001] direction measured by Stassis et al. are shown in Fig. 2. The temperature dependence of the phonon energy of the LO mode in the [0001] direction shows the anomalous behavior; i.e., the energies decrease with decreasing temperature. The anomalous behavior was explained by an instability toward the formation of a charge-density wave that originates from the band splitting near the Fermi level around the $A$ point in the first Brillouin zone by the lattice distortion. ${ }^{10,11}$ Recently Chen and Overhauser proposed that the anomalous behavior may be related to the longitudinally polarized spindensity wave with wave vector $\mathbf{q}=(2 \pi / c)[0001]{ }^{12}$ It is beyond our scope to include such effects in our present calculation, but it is interesting to note that the calculated phonon energies are increased by a few percent when we increase the

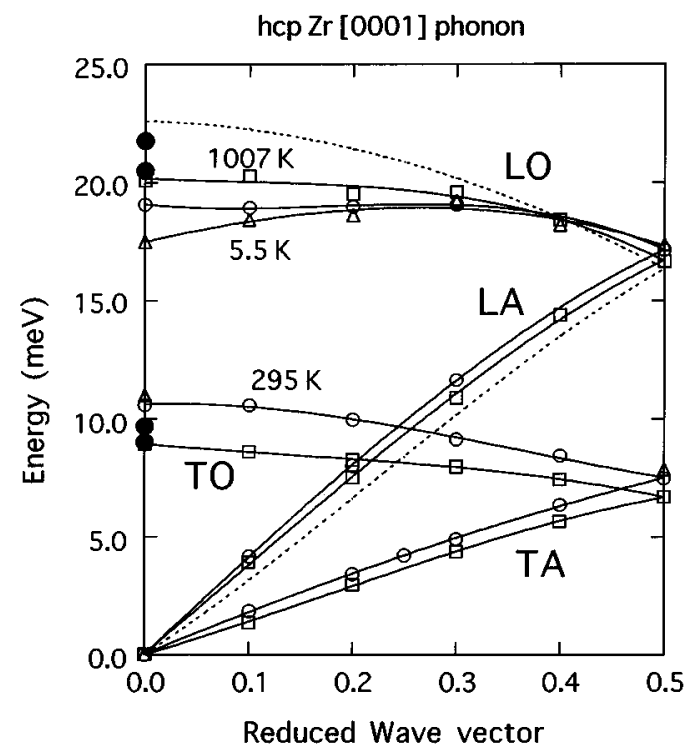

FIG. 2. Phonon dispersion curves of hcp zirconium along the [0001] direction measured by Stassis et al. (Ref. 9). The calculated zone-center LO and TO phonon energies are shown by closed circles. The dispersion of the longitudinal mode is calculated using the force constants for the fifth layer in the $\operatorname{Zr}(0001)$ slab with ten layers, and shown in the broken line.
Gaussian smearing for k-point summation from 0.05 to 0.5 $\mathrm{eV}$ to simulate the effect of smearing out the Fermi surface as temperature increases.

The zirconium surface system is represented by a supercell with repeated slabs of ten $\operatorname{Zr}(0001)$ layers, separated by a vacuum region with thickness corresponding to five layers. We used six special $\mathbf{k}$ points in the two-dimensional irreducible Brillouin zone for $\mathbf{k}$-point summation. The fully relaxed structure of this slab and its physical properties such as work function and surface energy, local density of states, electronic structure, and surface states and resonances were reported previously. ${ }^{8}$

The longitudinal vibrational modes along [0001] of the $\mathrm{Zr}(0001)$ slab can be studied as a one-dimensional linear chain; i.e., the (0001) plane moves as one unit in this mode and there is no buckling in the plane:

$$
\begin{aligned}
& m \ddot{u}_{i}=-\sum_{j \neq i} c_{i j}\left(u_{i}-u_{j}\right), \\
& u_{i}=A_{i} \exp \left[i\left(q z_{i}-\omega t\right)\right],
\end{aligned}
$$

where $m$ is the mass of a zirconium atom, $u_{i}$ is the displacement of the $i$ th plane from its equilibrium position $z_{i}, c_{i j}$ is the interlayer force constant between the $i-j$ plane, and $A_{i}$ is the vibrational amplitude of the $i$ th plane. In the previous work the surface relaxations $\Delta_{i j}\left\{\equiv\left[\left(z_{i}-z_{j}\right) /(\right.\right.$ interlayer distance in the bulk)]\} were found to be $-4.7,+1.2,+1.0$, -0.6 , and $+0.3 \%$ for $\Delta_{12}, \Delta_{23}, \Delta_{34}, \Delta_{45}$, and $\Delta_{56}$, respectively. ${ }^{8}$ For the interlayer force constants $c_{i j}=c_{j i}$ and there is an inversion center in this slab, then $c_{11-i, 11-j}=c_{i, j}$ for $i, j=1,2,3, \ldots, 9,10$.

Interlayer force constants are evaluated by computing the change of the forces (calculated via the Hellmann-Feynman theorem) upon small displacements of the atomic positions. The interlayer force constants up to the fourth nearestneighbor layer are obtained by giving a small displacement $\delta z$ of the two atoms of both sides of the slab in the $\pm z$ direction. The value of $\delta z$ is chosen to be 0.05 a.u., which is small enough that the anharmonic effect is negligible, while large enough that the numerical error from dividing by a small number can be neglected. Since inversion symmetry is imposed in the calculation, our analysis implicitly assumes that the interlayer force constants beyond the fourth nearest neighbor are small. This assumption may be justified because the interlayer forces determined from the experimental phonon dispersion curve in the [0001] direction become negligible beyond the fourth nearest neighbor. ${ }^{13,9}$ To determine the force constants uniquely, we use the following approximation: $c_{45} \simeq c_{56}$ and $c_{35} \simeq c_{46}$. The calculated force constants are shown in Table I. We find the magnitude of the interlayer force constants is decreased by one-tenth with increasing neighbor distance. The force constant $c_{12}$ between the surface and the second layer is larger than that in the bulk by $70 \%$.

Using the calculated interlayer force constants for the fifth layer (most inner layer) in the slab, we reproduce the phonon dispersion relation for the longitudinal mode in the [0001] direction. The result is shown as the broken line in Fig. 2. Except for the optic mode near the zone center, the dispersion relation is in agreement with experiment. 
TABLE I. Calculated force constants $c_{i j}$ for the $\operatorname{Zr}(0001)$ slabs with ten layers. Note that $c_{i j}=c_{j i}$ and $c_{i j}=c_{11-i, 11-j}$ for inversion symmetry. We made the approximation that $c_{i j}=0$ for $|i-j|>4, c_{45}=c_{56}$, and $c_{35}=c_{46}$. The force constants are given in the unit of $(\mathrm{N} / \mathrm{m})$.

\begin{tabular}{|c|c|c|c|c|c|c|c|c|c|c|}
\hline$i$ & 1 & 2 & 3 & 4 & 5 & 6 & 7 & 8 & 9 & 10 \\
\hline 1 & & 77.42 & 6.110 & 0.6694 & -0.9185 & 0 & 0 & 0 & 0 & 0 \\
\hline 2 & & & 42.44 & 4.265 & 1.923 & -0.7550 & 0 & 0 & 0 & 0 \\
\hline 3 & & & & 38.67 & 1.004 & -0.1712 & 0.1845 & 0 & 0 & 0 \\
\hline 4 & & & & & 44.86 & 1.004 & -0.0856 & 0.1845 & 0 & 0 \\
\hline 5 & & & & & & 44.86 & 1.004 & -0.1712 & -0.7550 & 0 \\
\hline
\end{tabular}

We calculated the dynamical matrix and obtained the phonon frequencies and their vibrational amplitudes for slabs with 10, 20, 50, and 100 layers, using the force constants determined for the fully relaxed ten-layer slab. The interlayer distances and the force constants for the layers below the fifth layer from the surface are assumed to be the same as the bulk values calculated. It has been reported by de Wette that the surface mode frequency, as well as the range of bulk modes, does not change significantly beyond a thickness of about 10 layers. ${ }^{3}$

As shown in Fig. 3, the most interesting features are the doubly degenerate localized states at about $23.6 \mathrm{meV}$, which split off from the bulk continuum. These split-off states are observed for all the slab thicknesses. They are about $1 \mathrm{meV}$ above the bulk continuum states, which have energies up to $22.6 \mathrm{meV}\left(\hbar \omega_{\text {bulk }}^{\max }\right)$. The depth dependence of the square of the amplitude of these localized mode $\left|A_{i}\right|^{2}$ is the same for all the slabs, and is shown in Fig. 4. Interestingly, these modes have the strongest amplitude at the second layer, and can thus be regarded as subsurface modes. As expected from the vibrational energies, the alternate (0001) layers oscillate

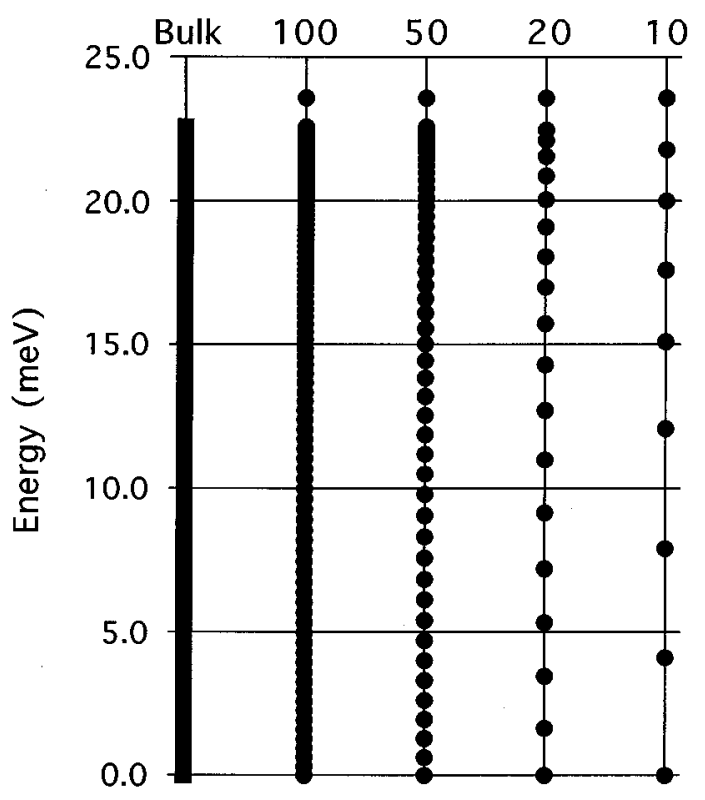

FIG. 3. Longitudinal phonon energies in the [0001] direction of the fully relaxed $\mathrm{Zr}(0001)$ slab with $10,20,50$, and 100 layers. The bulk phonon energies obtained from the dispersion curves (broken line in Fig. 1) are also shown. in opposite phases for these subsurface modes. If we use the force constants calculated for the bulk layer or those determined from the experimental bulk phonon dispersion, there is no surface-localized state above the $\hbar \omega_{\text {bulk }}^{\max }$ in the spectrum for the ideal $\operatorname{Zr}(0001)$ unrelaxed slabs with $10,20,50$, and 100 layers. Our results in the one-dimensional model correspond to the $\bar{\Gamma}$ vibrational states; then there are no split-off states below the continuum, i.e., the lowest vibrational energy of the $\bar{\Gamma}$ states is zero.

The density of states (DOS) obtained from the dispersion curve of the longitudinal phonon in the [0001] direction has a divergence at $\hbar \omega_{\text {bulk }}^{\max }$, since we are restricting ourselves to phonon modes in one direction only. The calculated DOS and the local density of states (LDOS) in the bulk layer of the slab with 100 layers show the same behavior, presented as histograms in Figs. 5(a) and 5(b). Here, the density of states $D(E)$ and local density of states $D(i, E)$ of the $i$ th layer are defined by

$$
D(E)=\sum_{\alpha} \delta\left(E-E_{\alpha}\right)
$$

$$
D(i, E)=\sum_{\alpha}\left|A_{i}(\alpha)\right|^{2} \delta\left(E-E_{\alpha}\right) .
$$

The LDOS of the surface layer $D(1, E)$ and that of the subsurface $D(2, E)$, however, do not have the divergence and

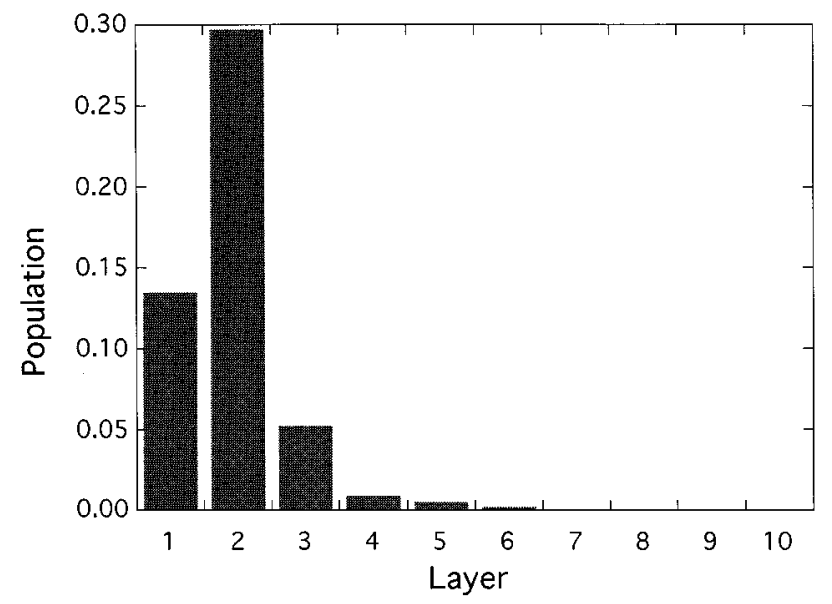

FIG. 4. Population of the amplitude of the localized phonon. Layers 1, 2, and 3 indicate the surface, subsurface, and third layers, respectively. 

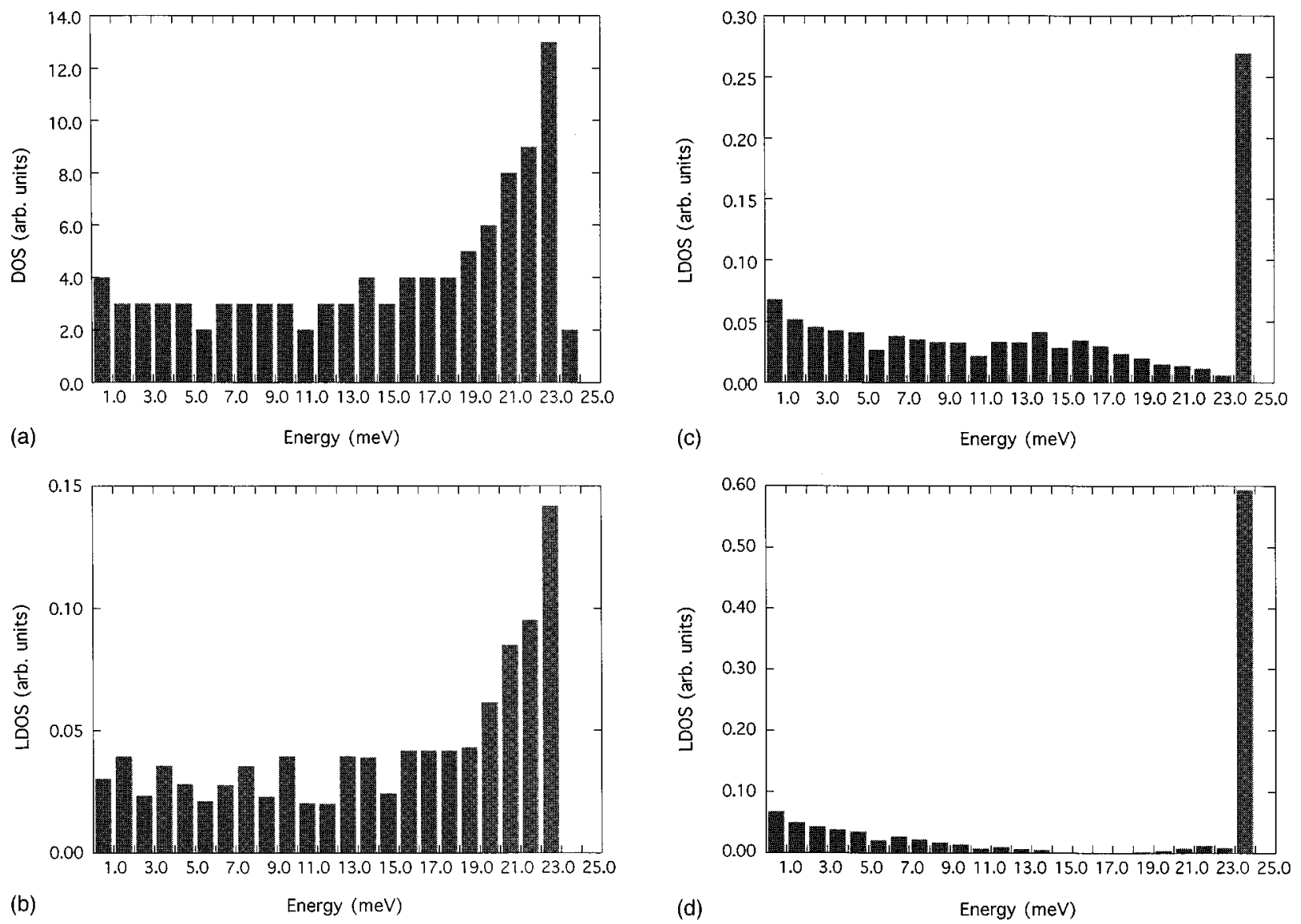

FIG. 5. Density of states (DOS) of the vibrational mode of the 100-layer zirconium slab in the [0001] direction. (a) Total DOS, (b) local density of states (LDOS) at the bulk 50th layer, (c) LDOS at the surface layer, and (d) LDOS at the subsurface layer.

have high DOS in the low-energy regions and in the energy region of the localized mode, as shown in Figs. 5(c) and 5(d).

Previous investigations have also suggested the existence of surface vibrational modes localized at the second layer of some transition-metal carbides such as $\mathrm{TiC}, \mathrm{ZrC}, \mathrm{HfC}, \mathrm{NbC}$, and $\mathrm{TaC}$. In these studies localization of phonon modes at the second layer has been suggested by empirical model calculations in order to explain the experimental phonon dispersion curves determined by HAS or HREELS. ${ }^{2}$ In monoatomic metal surfaces the subsurface localized modes above the bulk continuum for the reconstructed $\mathrm{W}(001)$ surface, ${ }^{15}$ the localized mode above the bulk continuum for the stepped $\mathrm{Pt}(111)$ surface, ${ }^{16}$ and some resonance modes localized at the subsurface for the $\mathrm{Ni}(100)$ surface ${ }^{17}$ have been reported. On the theoretical side the occurrence of the surface mode is well established in the study using the empirical model potential. ${ }^{18}$ However, to the best of our knowledge, our calculation is the first one in which phonon modes localized at the second layer of a surface system (while higher in frequency than bulk modes) are predicted by first-principles calculations, and given the reliability of first-principles results, there is good reason to believe that such modes do exist as predicted.

What is the physical origin of the second-layer localization? The increase in the restoring force of the second-layer atom may be the origin of the localization mode. From the calculated force constants shown in Table I, we estimate the restoring force of the $i$ th layer using $F_{i}=-\Sigma_{j \neq i} c_{i j} u_{i}$. The results are $F_{1}=-83.28 u_{1}, F_{2}=-125.29 u_{2}, F_{3}=$ $-88.24 u_{3}, F_{4}=-89.57 u_{4}$, and $F_{5}=-91.81 u_{5}$. The restoring force of the second layer is much greater than the others. The increase in the restoring force is originated from the stiffening of the force constants between the surface layer and the second layer as shown in Table I. In a previous calculation $^{8}$ we have shown that the increase of the electron density by the cohesion between the first layer and the second layer is greater than that in the bulk. The density increase, i.e., backbonding of the surface atom to the second layer, may be the origin of the increase in the force constant.

The ratio of the mean square displacement at the surface and at the bulk layer can be estimated from the phonon LDOS of the slabs. The mean square displacement in the surface normal due to thermal vibrations is ${ }^{14}$

$$
\left\langle u_{i}^{2}\right\rangle \propto \int d \omega D(i, \omega)[2\langle n(\omega)\rangle+1] / \omega .
$$

Here $\langle n(\omega)\rangle$ is the Bose-Einstein distribution function. For the slabs with 100 layers the surface-bulk ratio $\left\langle u_{s}^{2}\right\rangle /\left\langle u_{b}^{2}\right\rangle$ is found to be 1.9. Experimentally, the ratio of the mean square displacement of the surface layer to the bulk is approximately 2.0-2.5. The mean square displacement is obtained from the Debye-Waller factor analysis of LEED and EELFS 
measurements for the chemically equivalent $\mathrm{Ti}(0001)$ surface. ${ }^{5,6}$ Given that our DOS corresponds to one direction only (the $\bar{\Gamma}$ point in the surface Brillouin zone), our estimate of the mean square displacement is in reasonable agreement with the above experiment values. The increase in the ratio may be due to the increase of the LDOS at lower excitation energies at the surface layer.

In summary, we have calculated the energy and the amplitude of the longitudinal phonon modes in the surfacenormal direction of the $\operatorname{Zr}(0001)$ surface using the results from first-principles calculations. We found the existence of localized phonon vibrations at the subsurface region, with frequencies higher than bulk frequencies, and this mode is correlated with the stiffening of the interlayer force constant between the surface and the subsurface layer.

Ames Laboratory is operated for the U.S. Department of Energy by Iowa State University under Contract No. W-7405-ENG-82. Part of our work has been supported by the Director of Energy Research, Office of Basic Energy Sciences, including a grant of computer time on the Cray computers at the National Energy Research Supercomputer Center at Livermore.
${ }^{1}$ A. Zangwill, Physics at Surfaces (Cambridge University, Cambridge, 1988).

${ }^{2}$ C. Oshima, in Buturigaku Saizensen Vol. 30, edited by Y. Ootsuki (Kyoritu, Tokyo, 1992), pp. 175-250.

${ }^{3}$ J. P. Toennies, in Surface Phonons, edited by W. Kress and F. W. de Wette (Springer, Berlin, 1991), pp. 111-166; F. W. de Wette, ibid., pp. 67-109.

${ }^{4}$ S. J. Garrett, R. G. Egdell, and J. C. Rivière, J. Electron Spectrosc. Relat. Phenom. 54/55, 1065 (1990).

${ }^{5}$ P. R. Watson and J. Mischenko III, Surf. Sci. 186, 184 (1987).

${ }^{6}$ M. Kurahashi, M. Yamamoto, M. Mabuchi, and S. Naito, J. Phys. Condens. Matter 7, 37 (1995).

${ }^{7}$ K. M. Ho and K. P. Bohnen, Phys. Rev. Lett. 56, 934 (1986); Phys. Rev. B 38, 12897 (1988); J. Electron Spectrosc. Relat. Phenom. 54/55, 229 (1990); Y. Chen, S. Y. Tong, K. P. Bohnen, T. Rodach, and K. M. Ho, Phys. Rev. Lett. 70, 603 (1993).

${ }^{8}$ M. Yamamoto, C. T. Chan, and K. M. Ho, Phys. Rev. B 50, 7932 (1994).

${ }^{9}$ C. Stassis, J. Zarestky, D. Arch, O. D. MacMasters, and B. N. Harmon, Phys. Rev. B 18, 2632 (1978).
${ }^{10}$ S. H. Liu, C. Stassis, and K. M. Ho, Phys. Rev. B 24, 5093 (1981).

${ }^{11}$ N. Wakabayashi, R. H. Scherm, and H. G. Smith, Phys. Rev. B 25, 5122 (1982).

${ }^{12}$ X. M. Chen and A. W. Overhauser, Phys. Rev. B 42, 10873 (1990).

${ }^{13}$ C. Kittel, Introduction to Solid State Physics, 6th ed. (Wiley, New York, 1986).

${ }^{14}$ J. Callaway, Quantum Theory of the Solid State (Academic, San Diego, 1974), p. 49.

${ }^{15}$ J. P. Woods and J. L. Erskine, J. Vac. Sci. Technol. A 4, 1414 (1986); C. Z. Wang, A. Fasolino, and E. Tosatti, Phys. Rev. Lett. 59, 1845 (1987); A. Fasolino and E. Tosatti, Phys. Rev. B 35, 4264 (1987).

${ }^{16}$ H. Ibach and D. Bruchman, Phys. Rev. Lett. 41, 958 (1978).

${ }^{17}$ M. Rocca, S. Lehwald, H. Ibach, and T. S. Rahman, Surf. Sci. 171, 632 (1986).

${ }^{18}$ R. E. Allen, G. P. Alldredge, and F. W. de Wette, Phys. Rev. B 4, 1648 (1971); 4, 1661 (1971); S. W. Musser and K. H. Rieder, Phys. Rev. B 2, 3034 (1970). 